\title{
Estudo da Influência da precipitação de fases deletérias na resistência à corrosão por ensaio de polarização anódica cíclica em aços inoxidáveis superduplex
}

\author{
Study of deleterious phases precipitation influence \\ on corrosion resistance by cyclic anodic polarization \\ tests in superduplex stainless steels
}

\author{
Juan Manuel Pardal ${ }^{1}$, Sergio Souto Maior Tavares ${ }^{1}$, \\ Maria da Penha Cindra Fonseca ${ }^{1}$, João Vitor Silva Matias ${ }^{1}$ \\ Humberto Nogueira Farneze ${ }^{1}$, Eduardo Ariel Ponzio ${ }^{2}$
}

\footnotetext{
${ }^{1}$ Universidade Federal Fluminense (UFF) - Programas de Pós Graduação em Engenharia Mecânica (PGMEC) e Montagem Industrial - Rua Passo da Pátria 156, CEP 24210-240, São Domingos, Niterói-RJ, Brasil. e-mail:juanpardal@vm.uff.br, ssmtavares@terra.com.br, mcindra@vm.uff.br, jmatias@id.uff.br, humbertofarneze@gmail.com

${ }^{2}$ Universidade Federal Fluminense (UFF) - Departamento de Físico-Química. Programa de Pós-Graduação em Química Rua Outeiro de São João Batista s/n - CEP 24020-141 - Niterói / Brasil. e-mail: eaponzio@vm.uff.br
}

\section{RESUMO}

A influência da precipitação de fases deletérias na resistência à corrosão em dois aços inoxidáveis superduplex (AISD) UNS S32750 com diferentes tamanhos de grão foi investigada. Foram realizados tratamentos isotérmicos de modo a introduzir na microestrutura diversas quantidades de fases deletérias, tais como chi, sigma, austenita secundária e nitretos de cromo. Nestas condições foram efetuados ensaios de corrosão por polarização anódica cíclica. Estudos por microscopias ótica (MO) e eletrônica de varredura (MEV) foram realizados para caracterizar as fases precipitadas e a sua cinética de precipitação. Os resultados permitem correlacionar os valores obtidos pelos ensaios de polarização com o percentual de fases deletérias precipitadas para diferentes condições de tratamento em AISD com composições similares, mas com diferentes tamanhos de grão. Observou-se ainda que o tamanho de grão e a temperatura de envelhecimento influem na cinética de precipitação, na morfologia e no tamanho das fases deletérias precipitadas, incidindo na resistência à corrosão por pites dos AISD.

Palavras-chave: Aços inoxidáveis superduplex, Resistência à corrosão, Ensaio de polarização anódica cíclica.

\begin{abstract}
The influence of deleterious phases precipitation in corrosion resistance was investigated in two superduplex stainless steel (SDSS) UNS S32750 with different grain sizes. Isothermal treatments were performed in order to introduce various quantities of deleterious phases, such as chi, sigma, secondary austenite and chromium nitrides. Under these conditions corrosion tests were carried out by cyclic anodic polarization method. Studies by light optical microscopy (LOM) and scanning electron microscopy (SEM) were performed to characterize the precipitated phases and the precipitation kinetics. The results allow to correlate the values obtained by polarization tests with the percentage of deleterious phases precipitates to the different conditions of treatments of SDSS with similar compositions, but with different grain sizes. It was observed that the grain sizes and aging temperature influence the kinetics of precipitation, morphology and size of deleterious phases. These features affect the corrosion resistance of SDSS.
\end{abstract}

Keywords: Superduplex stainless steel, Corrosion resistance, Cyclic anodic polarization tests. 


\section{INTRODUÇÃO}

Os aços inoxidáveis duplex (AID) e superduplex (AISD) são materiais que aliam uma alta resistência mecânica com uma elevada resistência à corrosão por pites. Estas características são devidas à sua fina microestrutura bifásica composta por quantidades similares de austenita $(\gamma)$ e ferrita $(\delta)$ e aos elementos de liga $\mathrm{Cr}$, Mo e $\mathrm{N}$ [1]. No entanto, em faixas de temperaturas típicas de alguns processos de fabricação mecânica pode ocorrer a precipitação de fases deletérias, tais como, chi $(\chi)$, nitretos de $\operatorname{cromo}\left(\mathrm{Cr}_{2} \mathrm{~N}\right)$ e, principalmente, a sigma $(\sigma)$. Deste modo, foram realizados tratamentos isotérmicos, em AISD com diferentes tamanho de grão, com o intuito de introduzir na microestrutura diversas quantidades de fases deletérias. Estes compostos precipitados interagem em detrimento da resistência mecânica [2] e a resistência à corrosão da liga [3]. Entretanto, ocorre também a precipitação de austenita secundária $\left(\gamma_{2}\right)$ de baixa resistência à corrosão como consequência da decomposição eutetóide da ferrita $\left(\delta \rightarrow \sigma+\gamma_{2}\right)$, além da precipitação de outros tipos de $\gamma_{2}$ nas temperaturas de $800{ }^{\circ} \mathrm{C}$ a $950{ }^{\circ} \mathrm{C}$ [4]. O intervalo de temperaturas e cinética na precipitação de compostos deletérios é função da composição química do material [5] e do tamanho de grão da microestrutura [] $]$.

ANGELINI et al. [7] efetuaram ensaios de polarização cíclica, em um AISD, à temperatura ambiente em uma solução desaerada contendo $3,5 \%$ em peso de $\mathrm{NaCl}$. Nestas experiências observou-se que grandes tempos de tratamento aumentam a susceptibilidade da liga à corrosão localizada a $700{ }^{\circ} \mathrm{C}$ e $800{ }^{\circ} \mathrm{C}$, pois as curvas de polarização foram caracterizadas por um baixo potencial de pites $\left(E_{p}\right)$ e um extenso ciclo de histerese. Em contrapartida, não houve uma queda tão pronunciada, em comparação com a amostra solubilizada, das amostras tratadas a $900^{\circ} \mathrm{C}$ por diversos tempos de tratamento.

POTGIETER [8] apresenta resultados experimentais empregando a técnica de polarização cíclica, de acordo com as especificações da ASTM G61 [9]], para amostras de AID SAF 2205, tratadas a $850{ }^{\circ} \mathrm{C}$ em tempos de até 210 minutos. Os resultados indicam que a susceptibilidade à corrosão é maior nas amostras com maior conteúdo de fase $\sigma$. RAVINDRANATH e MALHOTRA [10] chegaram às mesmas conclusões para o mesmo material ensaiado por POTGIETER [8], empregando a técnica de polarização cíclica, em amostras tratadas no intervalo entre $700{ }^{\circ} \mathrm{C}$ e $900{ }^{\circ} \mathrm{C}$. Entretanto, POTGIETER [8] observou que somente 6\% desta fase causa uma considerável perda na resistência à corrosão do AID SAF 2205. SRIDHAR et al. [11] e, recentemente DOS SANTOS et al. [12] ressaltaram que há uma influência do mecanismo de formação de fase sigma no potencial de pites $\left(\mathrm{E}_{\mathrm{p}}\right)$ em AID.

$O$ presente trabalho visa avaliar a influência da precipitação de fases deletérias nos valores do $E_{p}$ para ensaios de polarização anódica cíclica a $60^{\circ} \mathrm{C}$ em AISD tratados termicamente com composições químicas similares, mas com diferente tamanho de grão. A morfologia e a fração de fases precipitadas em diferentes temperaturas influenciam, não somente com os valores do $E_{p}$, como também do potencial de repassivação $\left(\mathrm{E}_{\text {гр }}\right)$ e da densidade de corrente máxima na região ativa $\left(\mathrm{I}_{\text {máx }}\right)$ da curva de polarização cujo maior valor deste parâmetro indica uma maior degradação do material em termos de áreas empobrecidas em Cr e Mo para uma determinada temperatura de ensaio [13]

\section{MATERIAIS E MÉTODOS}

Neste trabalho, foram estudados dois tipos de AISD atendendo à designação UNS S32750, sendo os mesmos identificados como "SD-A" e "SD-B". A Tabela 1 apresenta a composição química dos materiais de estudo, observando-se composições similares em ambos os casos.

Tabela 1: Composição química dos AISD analisados.

\begin{tabular}{c|c|c|c|c|c|c|c|c|c|c|c}
\hline \multirow{2}{*}{ Material } & \multicolumn{10}{c}{ Composição química (\% em peso). Fe balanço. } \\
\cline { 2 - 13 } & $\mathbf{C r}$ & $\mathbf{N i}$ & $\mathbf{M o}$ & $\mathbf{M n}$ & $\mathbf{S i}$ & $\mathbf{N}$ & $\mathbf{C u}$ & $\mathbf{W}$ & $\mathbf{C}$ & $\mathbf{P}$ & $\mathbf{S}$ \\
\hline SD-A & 24,57 & 6,68 & 3,75 & 0,83 & 0,34 & 0,28 & 0,25 & --- & 0,02 & 0,03 & 0,000 \\
\hline SD-B & 24,80 & 6,75 & 3,79 & 0,78 & 0,54 & 0,27 & 0,10 & 0,04 & 0,02 & 0,03 & 0,001 \\
\hline
\end{tabular}

Amostras de aproximadamente 15 x 10 x 4,5 mm foram retiradas dos materiais na condição como recebidos (CR) para estudo. Posteriormente, as amostras foram tratadas isotermicamente a 800/850/900 e $950{ }^{\circ} \mathrm{C}$ em tempos de 15 e 60 minutos, utilizando água como meio de resfriamento. 
O estudo metalográfico foi realizado em um microscópio ótico (MO) modelo NEOPHOT 32, avaliando a superfície das amostras tratadas termicamente As quantificações das fases $\delta$ e $\gamma$, no estado solubilizado, pela utilização do reagente Beraha, assim como das fases deletérias, através da aplicação do ataque eletrolítico $\mathrm{KOH}$, foram realizadas através de uso de software específico [14], com média de 20 imagens por condição de tratamento e com diferentes aumentos metalográficos.

Para a realização de estudos específicos na determinação do tamanho de grão (TG) das fases $\delta$ e $\gamma$ foi usado um MEV da marca PHILIPS ${ }^{\circledR}$, modelo XL30 mediante uso da técnica de difração de elétrons retroespalhados (EBSD). A Tabela 2 sumariza as proporções de fases, assim como tamanhos de grão correspondentes à ferrita $(\delta)$ e austenita $(\gamma)$ presentes na condição solubilizada para cada material [6]. Mesmo que a composição química de ambos os materiais sejam similares, as diferenças das fases e tamanhos de grãos precipitados entre o "SD-A" e "SD-B" são atribuídos às distintas formas de processamento destes materiais segundo as normas atendidas em cada material. A Figura 1 mostra as microestruturas dos dois materiais no estado solubilizado, antes de serem efetuados os tratamentos térmicos de envelhecimento.

Tabela 2: Proporções de fases e tamanhos de grão (TG) dos AISD estudados.

\begin{tabular}{|c|c|c|c|c|c|c|}
\hline \multirow{3}{*}{ Material } & \multicolumn{3}{|c|}{ Ferrita $(\delta)$} & \multicolumn{3}{|c|}{ Austenita $(\gamma)$} \\
\hline & \multirow{2}{*}{$\begin{array}{c}\text { Fração vol. } \\
(\%)\end{array}$} & \multicolumn{2}{|c|}{ Tamanho de Grão } & \multirow{2}{*}{$\begin{array}{c}\text { Fração vol. } \\
\text { (\%) }\end{array}$} & \multicolumn{2}{|c|}{ Tamanho de Grão } \\
\hline & & $(\mu \mathrm{m})$ & ASTM $n^{\circ}$ & & $(\mu \mathrm{m})$ & ASTM $n^{\circ}$ \\
\hline SD-A & $55,05 \pm 1,90$ & 29,42 & 7,2 & $44,95 \pm 1,90$ & 24,75 & 7,7 \\
\hline SD-B & $49,95 \pm 1,26$ & 132,36 & 2,8 & $50,05 \pm 1,26$ & 138,32 & 2,7 \\
\hline
\end{tabular}
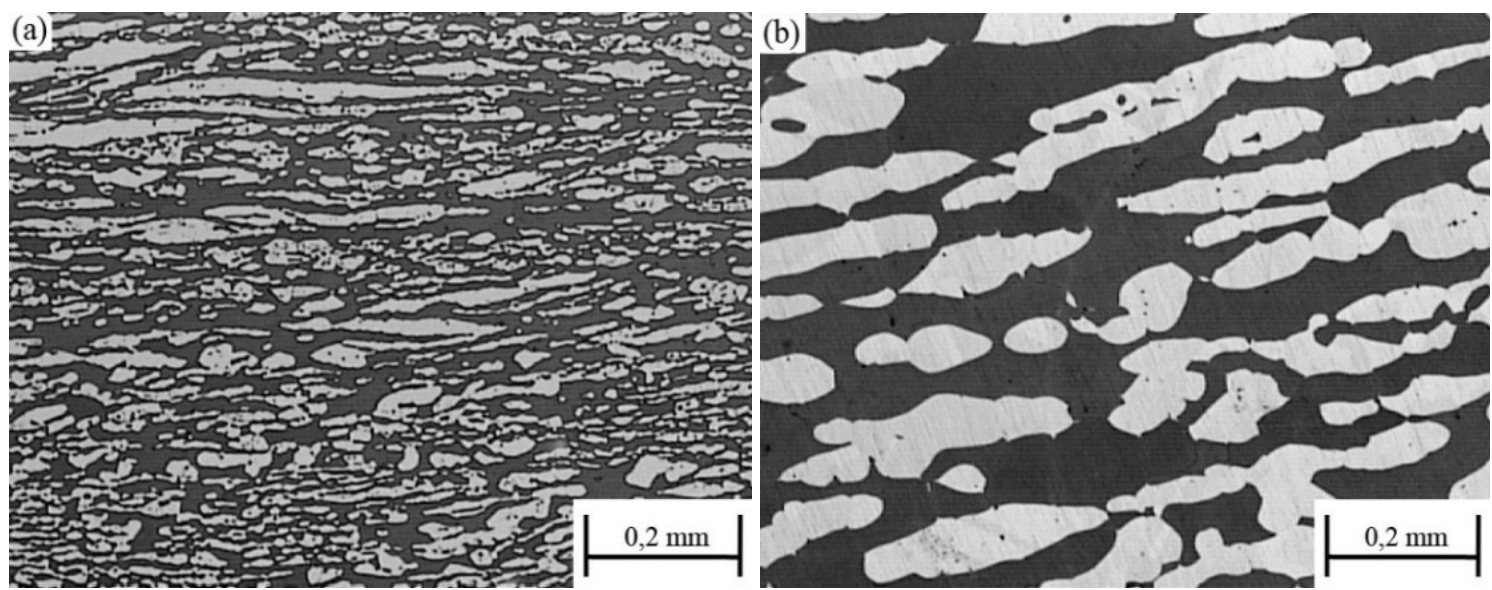

Figura 1: Materiais como recebidos (CR) em estado solubilizado. SD-A e SD-B, respectivamente.

Os ensaios de polarização cíclica foram efetuados em um potenciostato galvanostato $\mu$ Autolab® Type III. Os eletrodos de trabalho foram fabricados através da fixação da amostra a um fio rígido de Cu. Em seguida, estes eletrodos foram embutidos em resina de cura a frio. A superfície da cada amostra em contato com o eletrólito foi preparada pelo processo metalográfico convencional, empregando-se como etapa final o polimento com alumina em suspensão aquosa de $0,1 \mu \mathrm{m}$. De modo a evitar corrosão por frestas, as laterais e vértices do eletrodo em contato com a resina foram recobertas com esmalte incolor. Os ensaios por esta técnica foram realizados em uma solução modificada de $1 \mathrm{M} \mathrm{H}_{2} \mathrm{SO}_{4}+1 \mathrm{M} \mathrm{NaCl}$ à temperatura de $60{ }^{\circ} \mathrm{C}$, segundo procedimento semelhante ao da norma ASTM G61 [9]]. Para a determinação do potencial de corrosão, os eletrodos ficaram expostos durante aproximadamente 30 minutos na solução indicada. Logo, a polarização foi realizada com uma taxa de varredura de $1 \mathrm{mV}_{\mathrm{SCE}} / \mathrm{s}$, a partir do potencial de corrosão determinado. O potencial foi revertido ao ser atingida a densidade de corrente $\left(\delta_{\mathrm{i}}\right)$ de $5 \mathrm{~mA} / \mathrm{cm}^{2}$. Todos os ensaios foram efetuados com aeração natural. Devido à difícil obtenção do potencial de pites $\left(\mathrm{E}_{\mathrm{p}}\right)$ foi adotado o método proposto por NASCIMENTO et al. [15] para a determinação precisa do mesmo. A Figura 2 mostra como exemplo, a obtenção do $E_{P}$ pela projeção das retas tangentes à curva próxima ao rompimento da camada passiva na condição tratada a $850{ }^{\circ} \mathrm{C}$ por 15 minutos do SD-A. No entanto, outras variáveis importantes foram determinadas através da representação gráfica das curvas de polarização, tal como exibe a 
figura mencionada. Os valores aproximados do potencial de repassivação $\left(E_{r p}\right)$ e da densidade de corrente máxima na região ativa $\left(\mathrm{I}_{\text {máx }}\right)$ foram obtidos em cada condição, de modo de avaliar o grau de degradação nas diferentes condições de tratamento.

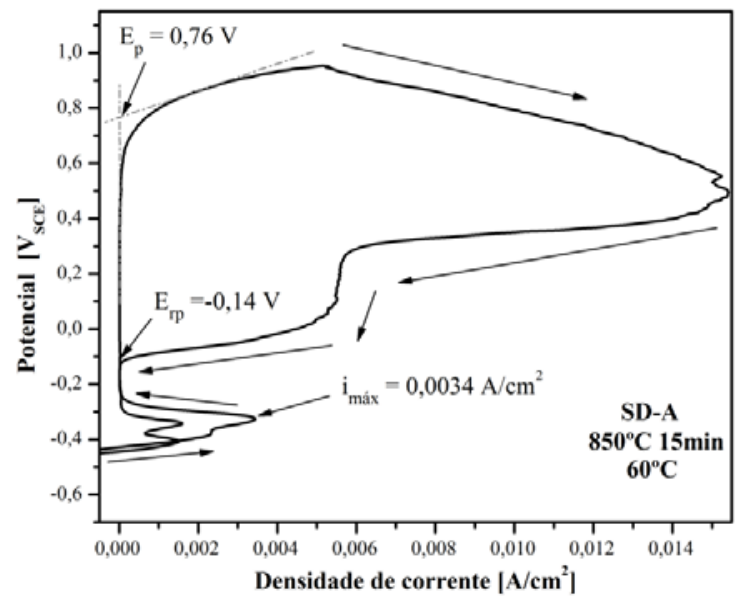

Figura 2: Determinação do $\mathrm{E}_{\mathrm{p}}, \mathrm{E}_{\mathrm{rp}}$ e $\mathrm{i}_{\text {máx }}$ para o material SD-A tratado isotermicamente a $850{ }^{\circ} \mathrm{C}$ por 15 minutos.

Após os ensaios de polarização cíclica as superfícies das amostras foram avaliadas por microscopias ótica (MO) e eletrônica de varredura (MEV). Para conseguir uma ótima visualização no microscópio, um leve polimento com alumina em suspensão de 0,1 $\mu \mathrm{m}$ foi realizado previamente, em todos os casos, para evidenciar na microestrutura pites e locais preferenciais de corrosão. Cabe destacar que, as imagens obtidas no MEV foram realizadas em um microscópio eletrônico JEOL 6460LV no modo de elétrons secundários.

\section{RESULTADOS}

A Figura 3 exibe as curvas de polarização cíclica, levantadas pela metodologia descrita, dos materiais SD-A e SD-B como recebidos (CR). A semelhança entre as curvas pode ser atribuída à pequena diferença nas composições químicas dos materiais. Cabe salientar que ambos os materiais estão isentos de fases deletérias pela aplicação do reagente de $\mathrm{KOH}$. Os valores característicos de $\mathrm{E}_{\mathrm{p}}, \mathrm{E}_{\mathrm{rp}}$ e $\mathrm{I}_{\text {máx }}$ dos materiais $\mathrm{CR}$ são apresentados na Tabela 3, embora os valores de potenciais de pites $\left(\mathrm{E}_{\mathrm{p}}\right)$ obtidos, próximos de 1,0 $\mathrm{V}_{\mathrm{SCE}}$, com repassivação total do ciclo e, sem presença de histerese, indiquem provavelmente à eletrólise do eletrólito nestas condições. Sendo assim foi considerado que, os valores de $E_{p}$ e $E_{r p}$ levantados que exibam comportamento como aqueles apresentados na Figura 3, tenham valores mínimos de $\mathrm{E}_{\mathrm{p}}$ e $\mathrm{E}_{\mathrm{rp}}$ obtidos pela metodologia descrita neste trabalho.

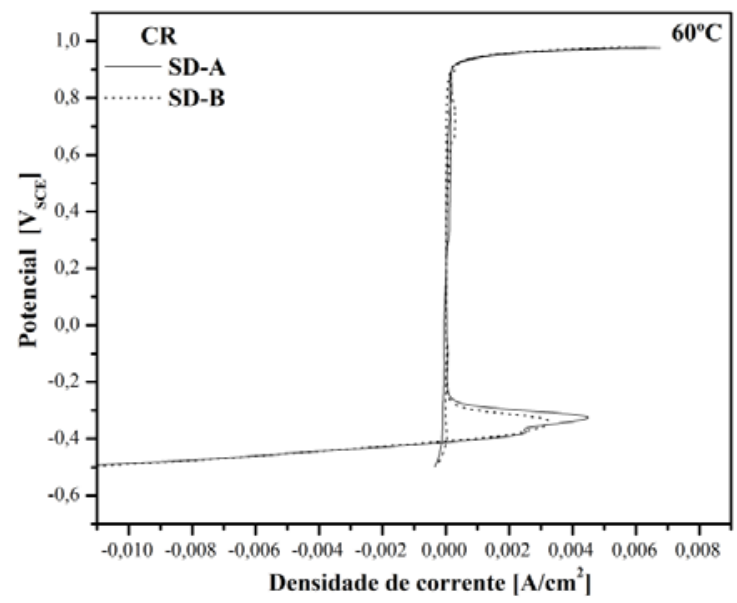

Figura 3: Curvas de polarização cíclica anódica dos materiais como recebidos. 
Tabela 3: Valores característicos de $\mathrm{E}_{\mathrm{p}}, \mathrm{E}_{\mathrm{rp}}$ e $\mathrm{I}_{\text {máx }}$, extraídos das curvas de polarização da Figura 2 para as amostras SDA e SD-B como recebidas.

\begin{tabular}{c|c|c|c|c|c|c}
\hline \multirow{2}{*}{ Condição } & \multicolumn{3}{|c|}{ SD-A } & \multicolumn{3}{c}{ SD-B } \\
\cline { 2 - 7 } & $E_{\mathrm{p}}\left(\mathrm{V}_{\text {SCE }}\right)$ & $\mathrm{E}_{\mathrm{rp}}\left(\mathrm{V}_{\text {SCE }}\right)$ & $\mathrm{I}_{\text {máx }}\left(\mathbf{A} / \mathrm{cm}^{2}\right)$ & $\mathrm{E}_{\mathrm{p}}(\mathrm{V})$ & $\mathrm{E}_{\mathrm{rp}}\left(\mathrm{V}_{\text {SCE }}\right)$ & $\mathrm{I}_{\text {máx }}\left(\mathbf{A} / \mathbf{c m}^{2}\right)$ \\
\hline CR & $>0,94$ & $>0,98$ & 0,0045 & $>0,94$ & $>0,98$ & 0,0032 \\
\hline
\end{tabular}

As Figuras 4-7 apresentam as curvas de polarização anódica cíclica para as condições tratadas isotermicamente a $800^{\circ} \mathrm{C}, 850^{\circ} \mathrm{C}, 900^{\circ} \mathrm{C}$ e $950^{\circ} \mathrm{C}$ em tempos de 15 e 60 minutos para os AISD SD-A e SDB. A Tabela 4 exibe os valores característicos extraídos de cada ciclo. Os valores percentuais das fases deletérias (FD) precipitadas foram acrescentados, nesta tabela, para comparação. Neste trabalho, ficou evidente que, nestes tratamentos, o maior tamanho de grão do SD-B confere uma maior resistência à corrosão por pites em relação ao SD-A, como consequência de uma menor cinética de precipitação de fases deletérias. Estudos realizados, nestes materiais, pela técnica de polarização eletroquímica de reativação cíclica (PERC) tiveram resultados similares [16].

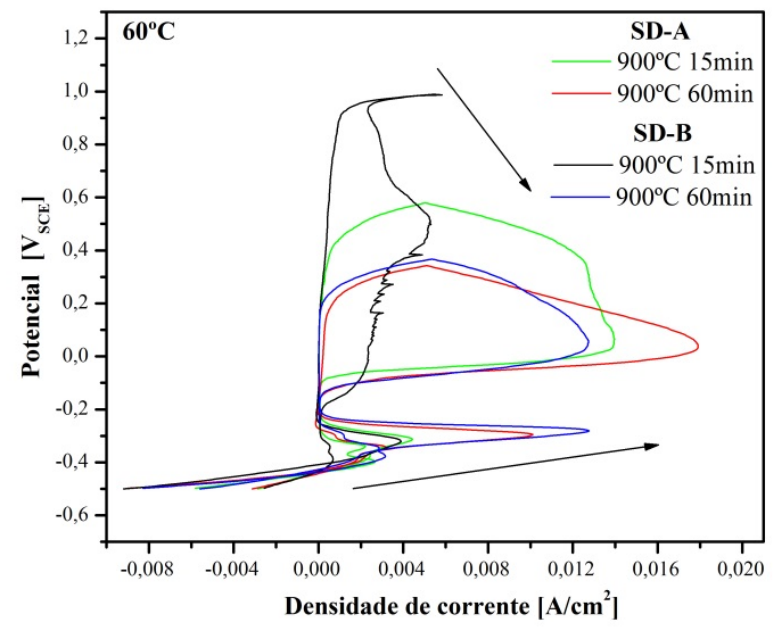

Figura 4: Ciclos de polarização anódica das amostras tratadas isotermicamente a $800{ }^{\circ} \mathrm{C}$ por 15 e 60 minutos nos AISD SD-A e SD-B.

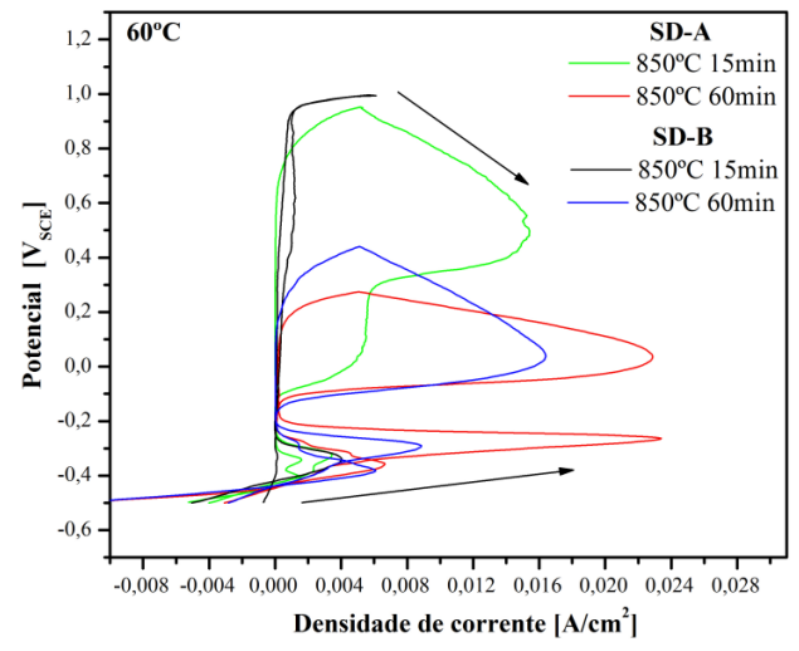


PARDAL, J.M.; TAVARES, S.S.M.; CINDRA FONSECA, M.P.; MATIAS, J.V.S., revista Matéria, v.20, n.2, pp. 374 - 383 , 2015.

Figura 5: Ciclos de polarização anódica das amostras tratadas isotermicamente a $850{ }^{\circ} \mathrm{C}$ por 15 e 60 minutos nos AISD SD-A e SD-B.

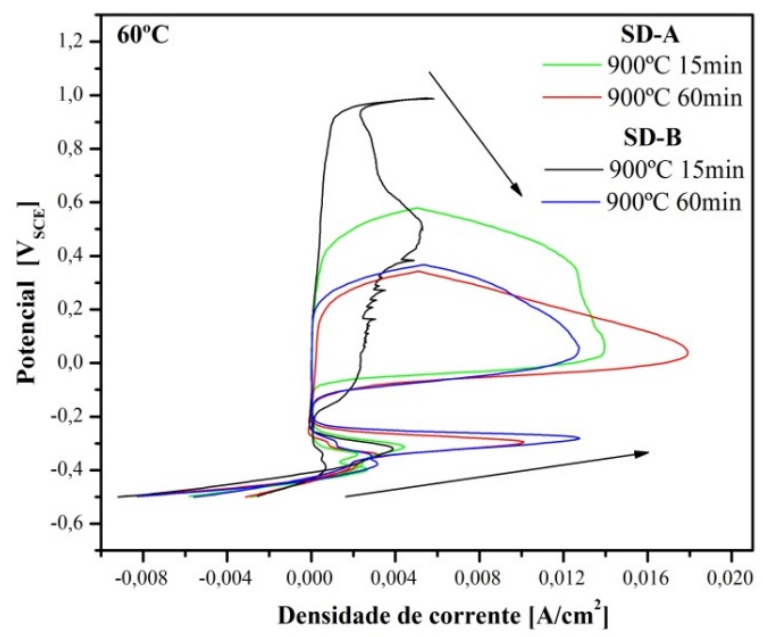

Figura 6: Ciclos de polarização anódica das amostras tratadas isotermicamente a $900{ }^{\circ} \mathrm{C}$ por 15 e 60 minutos nos AISD SD-A e SD-B.

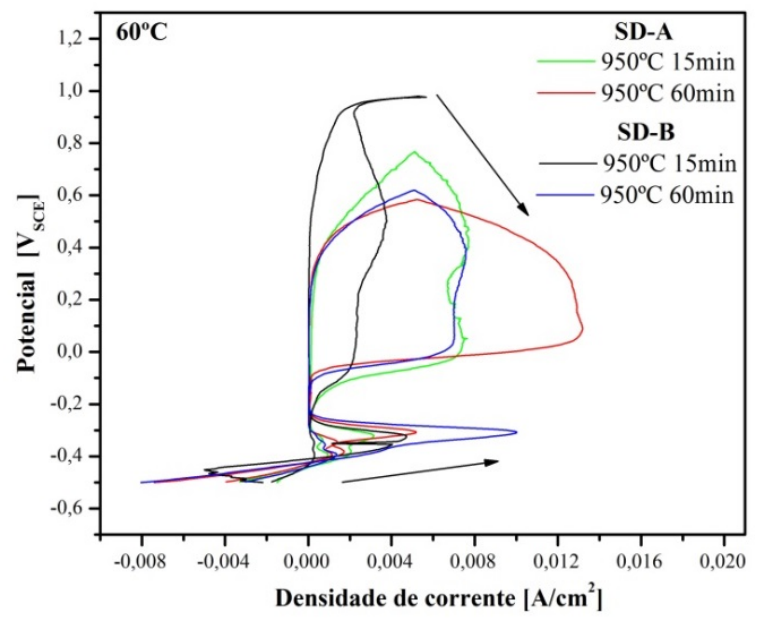

Figura 7: Ciclos de polarização anódica das amostras tratadas isotermicamente a $950{ }^{\circ} \mathrm{C}$ por 15 e 60 minutos nos AISD SD-A e SD-B. 
Tabela 4: Valores característicos de $\mathrm{E}_{\mathrm{p}}$, $\mathrm{E}_{\mathrm{rp}}$ e $\mathrm{I}_{\text {máx }}$, extraídos das curvas de polarização das Figuras 3-6 para as diversas condições de tratamento isotérmico nos AISD estudados. Os valores percentuais das FD precipitadas foram acrescentados para comparação.

\begin{tabular}{|c|c|c|c|c|c|c|c|c|c|}
\hline \multirow{2}{*}{\multicolumn{2}{|c|}{ Condição }} & \multicolumn{4}{|c|}{ SD-A } & \multicolumn{4}{|c|}{ SD-B } \\
\hline & & \multirow{2}{*}{$\begin{array}{c}\begin{array}{c}\mathbf{E}_{\mathrm{p}} \\
\left(\mathbf{V}_{\mathrm{SCE}}\right)\end{array} \\
0,94\end{array}$} & \multirow{2}{*}{$\begin{array}{c}\begin{array}{c}\mathbf{E}_{\text {rp }} \\
\left(\mathbf{V}_{\text {SCE }}\right)\end{array} \\
-0,23^{*}\end{array}$} & \multirow{2}{*}{$\begin{array}{c}\begin{array}{c}I_{\text {máx }} \\
\left(\mathbf{A} / \mathbf{c m}^{2}\right)\end{array} \\
0,0038\end{array}$} & \multirow{2}{*}{$\begin{array}{c}\text { FD } \\
(\%)\end{array}$} & \multirow{2}{*}{ 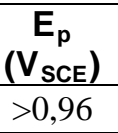 } & \multirow{2}{*}{$\begin{array}{l}\mathbf{E}_{\mathrm{rp}} \\
\left(\mathbf{V}_{\mathrm{SCE}}\right) \\
>1,00\end{array}$} & \multirow{2}{*}{$\begin{array}{c}\begin{array}{c}I_{\text {máx }} \\
\left(\mathbf{A} / \mathbf{c m}^{2}\right)\end{array} \\
0,0037\end{array}$} & \multirow{2}{*}{$\begin{array}{c}\begin{array}{c}\text { FD } \\
(\%)\end{array} \\
0,83 \pm 0,18\end{array}$} \\
\hline & $15 \mathrm{~min}$. & & & & & & & & \\
\hline $800^{\circ} \mathrm{C}$ & $60 \mathrm{~min}$. & 0,09 & $-0,15$ & 0,0311 & $25,99 \pm 0,72$ & 0,69 & $-0,19$ & 0,0063 & $7,75 \pm 0,62$ \\
\hline \multirow{2}{*}{$850^{\circ} \mathrm{C}$} & $15 \mathrm{~min}$. & 0,76 & $-0,14$ & 0,0034 & $12,06 \pm 1,68$ & 0,97 & $\begin{array}{l}-0,25 * \\
\end{array}$ & 0,0040 & $3,47 \pm 0,91$ \\
\hline & $60 \mathrm{~min}$. & 0,19 & $-0,15$ & 0,0232 & $38,03 \pm 1,34$ & 0,30 & $-0,19$ & 0,0089 & $31,02 \pm 1,36$ \\
\hline \multirow{2}{*}{$900^{\circ} \mathrm{C}$} & $15 \mathrm{~min}$. & 0,23 & $-0,21$ & 0,0044 & $16,42 \pm 0,84$ & 0,95 & $-0,25 *$ & 0,0038 & $4,62 \pm 0,82$ \\
\hline & $60 \mathrm{~min}$. & 0,25 & $-0,17$ & 0,0100 & $35,22 \pm 1,34$ & 0,27 & $-0,18$ & 0,0126 & $37,45 \pm 1,35$ \\
\hline \multirow{2}{*}{$950^{\circ} \mathrm{C}$} & $15 \mathrm{~min}$. & 0,43 & $-0,24$ & 0,0031 & $17,38 \pm 0,80$ & 0,94 & $-0,25 *$ & 0,0047 & $8,70 \pm 0,84$ \\
\hline & $60 \mathrm{~min}$. & 0,46 & $-0,22$ & 0,0051 & $27,52 \pm 0,58$ & 0,43 & $-0,18$ & 0,0100 & $29,73 \pm 1,38$ \\
\hline
\end{tabular}

* Casos onde houve uma repassivação parcial do ciclo.

Em todos os casos, as condições tratadas por 15 minutos no SD-B apresentaram um alto valor de $E_{p}$. Entretanto, foram notadas evidências de repassivação parcial, com a consequente formação de uma pequena histerese no ciclo, quanto maiores forem as temperaturas de tratamento. No caso do SD-A tratado por 15 minutos somente houve repassivação parcial para a amostra tratada a $800{ }^{\circ} \mathrm{C}$, enquanto isso, na mesma condição, para o SD-B houve repassivação total, sem a existência de histerese no ciclo, como mostra a Figura 4. Observou-se que o fenômeno de repassivação no AISD tratado isotermicamente por 15 minutos foi mais efetivo no material com maior tamanho de grão, provavelmente devido à menor quantidade de fase $\sigma$ precipitada.

Na condição de tratamento a $800{ }^{\circ} \mathrm{C}$ por 60 minutos se observa comparativamente uma maior resistência à corrosão por pites no SD-B em relação ao SD-A que, por sua vez, apresentou um valor elevado da $\mathrm{I}_{\text {máx }}$. Conforme mostrados na Tabela 4, os valores de $\mathrm{E}_{\mathrm{p}}$ e $\mathrm{I}_{\text {máx }}$ tendem a se aproximar, em ambos os materiais, em temperaturas maiores de tratamento por 60 minutos, destacando-se que a condições tratadas a 800 e $850{ }^{\circ} \mathrm{C}$ por 60 minutos no SD-A foram aquelas que apresentaram os menores valores de $\mathrm{E}_{\mathrm{p}}$ de todas as condições avaliadas. Para esta última condição, KOBAYASHI et al. [17], obtiveram resultados similares efetuando ensaios de polarização em diversos AID.

Por outro lado, em ambos os materiais, a condição tratada a $950{ }^{\circ} \mathrm{C}$ por 60 minutos apresentaram $\mathrm{E}_{\mathrm{p}}$ semelhantes, sendo estes de maior valor do que aqueles obtidos para as condições tratadas a 850 e $900{ }^{\circ} \mathrm{C}$ por 60 minutos. Neste sentido, as Figuras 8a e 8b exibem as características microestruturais obtidas por MO após os ensaios de polarização no SD-A tratado, por 850 e $950{ }^{\circ} \mathrm{C}$ por 60 minutos, respectivamente. Efetuando-se uma análise comparativa destas figuras se observa claramente a tendência, na maior temperatura de tratamento, da precipitação de placas mais grosseiras de fase $\sigma$ que podem ter sido oriundas tanto da decomposição lamelar divorciada eutetóide de ferrita $\left(\delta \rightarrow \sigma+\gamma_{2}\right)$ ou da precipitação pela transformação direta a partir da ferrita $(\delta \rightarrow \sigma)$ [13]. Ambas as transformações são consequência de variações nas taxas de nucleação e de difusão em relação às menores temperaturas de tratamento atribuindo-se deste modo uma morfologia mais maciça. As Figuras 9 e 10 mostram, mais claramente, este fenômeno através de imagens obtidas por MEV, com aumentos semelhantes, após ensaios de polarização no SD-B nas mesmas condições de tratamento descritas para o SD-A. Neste caso, se observa um severo ataque na quase extinta fase ferrita da amostra tratada a $850{ }^{\circ} \mathrm{C}$, ao passo que na condição de $950{ }^{\circ} \mathrm{C}$ se observam grandes placas de $\sigma$ oriundas da fase ferrita, podendo nestes sítios existir um processo de redifusão de certos elementos de liga que propiciem a melhoria parcial de resistência à corrosão por pites da liga. Além destes fatos, vale a pena ressaltar que nesta temperatura também se manifesta uma precipitação mais intensa de $\gamma_{2}$, formada independentemente ou a partir de nitretos, tendo uma maior resistência à corrosão em relação àquela $\gamma_{2}$ precipitada como produto da decomposição eutetóide da ferrita [4]. Principalmente, por estes motivos, obteve-se um maior valor $\mathrm{E}_{\mathrm{p}}$ nas amostras tratadas a $950{ }^{\circ} \mathrm{C}$ por 60 minutos em relação à condição tratada a $850{ }^{\circ} \mathrm{C}$ por 60 minutos. 

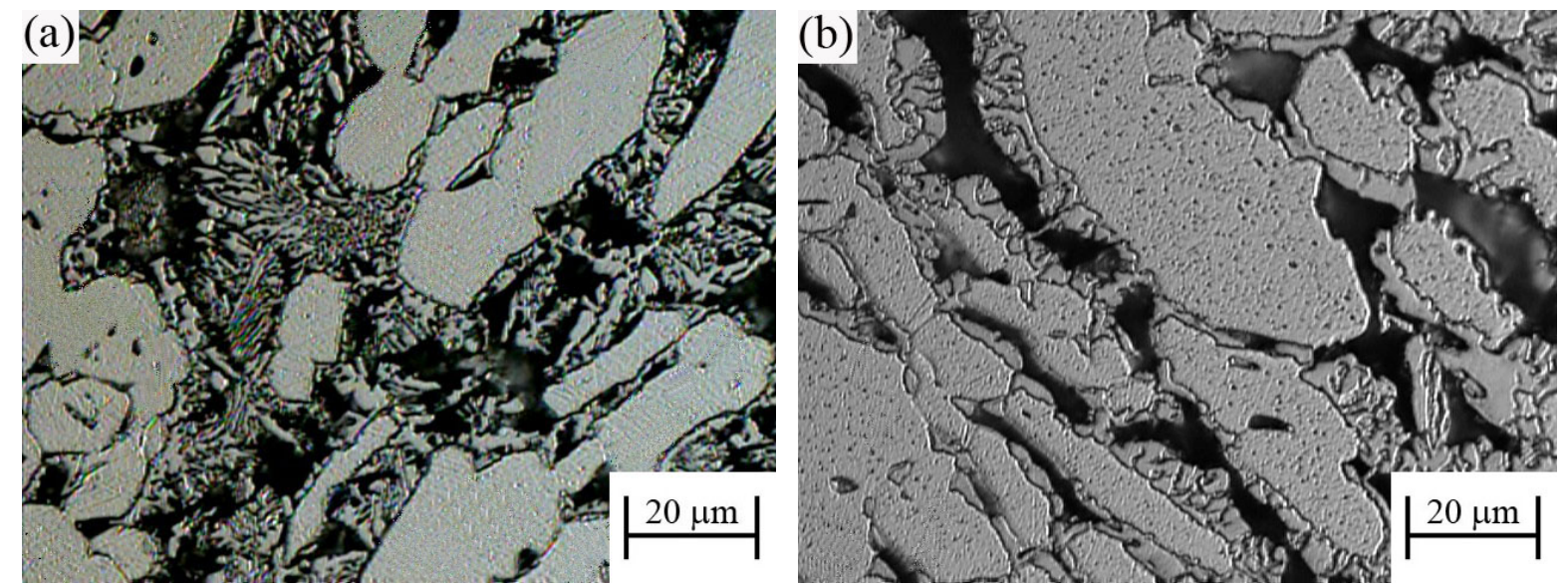

Figura 8: Microestruturas obtidas por MO após os ensaios de polarização em diversas condições de tratamento isotérmico no SD-A: (a) $850{ }^{\circ} \mathrm{C} 60$ minutos. (b) $950{ }^{\circ} \mathrm{C} 60$ minutos.
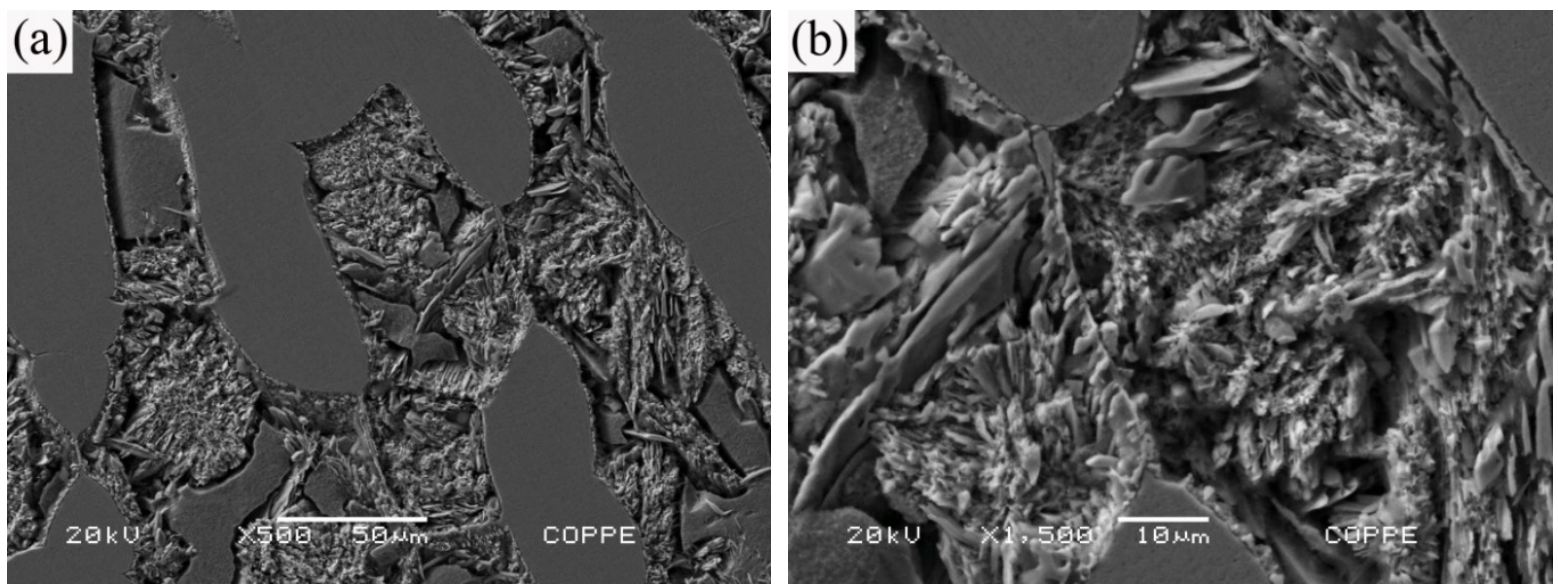

Figura 9: Análise da microestrutura obtida por MEV em determinada região após ensaios de polarização no SD-B tratado a $850^{\circ} \mathrm{C}$ por 60 minutos: (a) 500X (b) $1500 \mathrm{X}$.

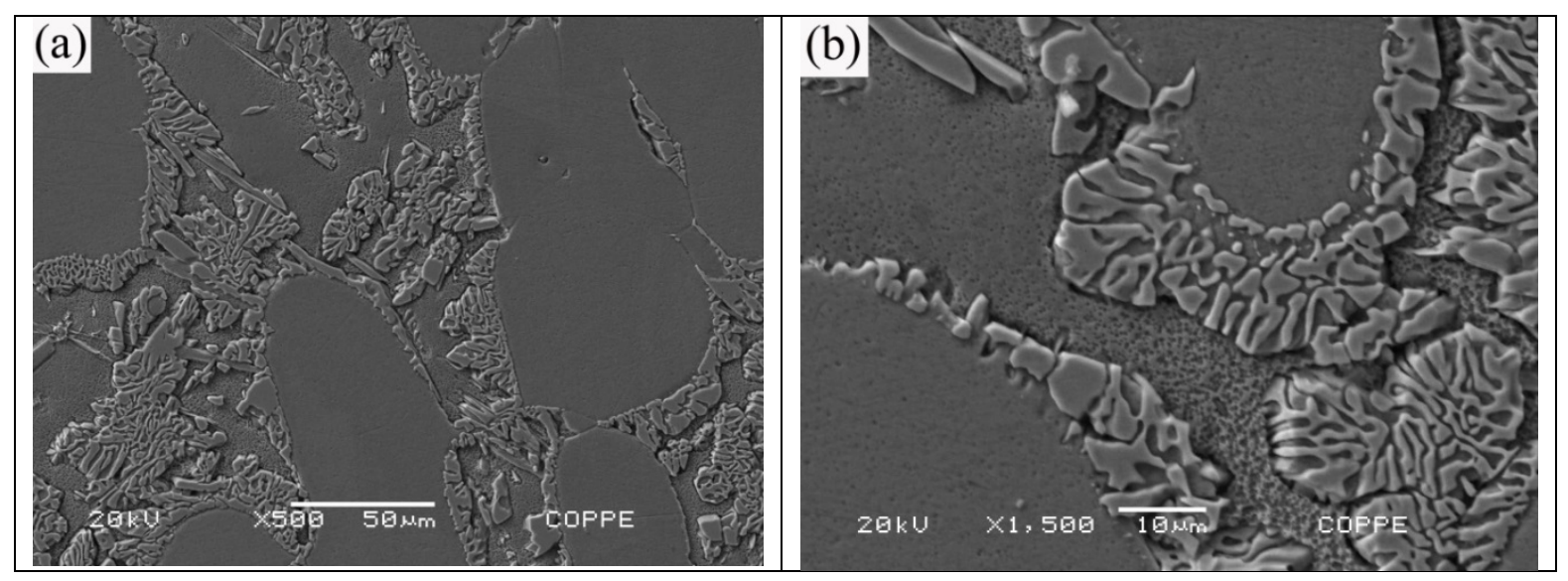

Figura 10: Análise da microestrutura obtida por MEV em determinada região após ensaios de polarização no SD-B tratado a $950^{\circ} \mathrm{C}$ por 60 minutos: (a) 500X (b) $1500 \mathrm{X}$. 
Em seu trabalho, KOBAYASHI et al. [17] salientam que a presença de finas lamelas de fase $\sigma \mathrm{e} \gamma_{2}$, produto da decomposição da $\delta$ são locais muito susceptíveis de quebra do filme da película passiva permitindo, deste modo, que uma corrosão seletiva desta fase prossiga facilmente. Neste sentido, a Figura 10 mostra uma determinada região da microestrutura tratada isotermicamente a $950^{\circ} \mathrm{C}$ por 15 minutos no SD-A. As imagens desta figura conseguem focalizar, tanto as regiões de maior resistência à corrosão apresentadas na Figura 11a, quanto aquelas susceptíveis à corrosão mostrada na Figura 11b. Na Figura 11b é observada uma área com microestrutura lamelar muito provavelmente resultante da decomposição lamelar da $\sigma$ sob estas condições de tratamento.
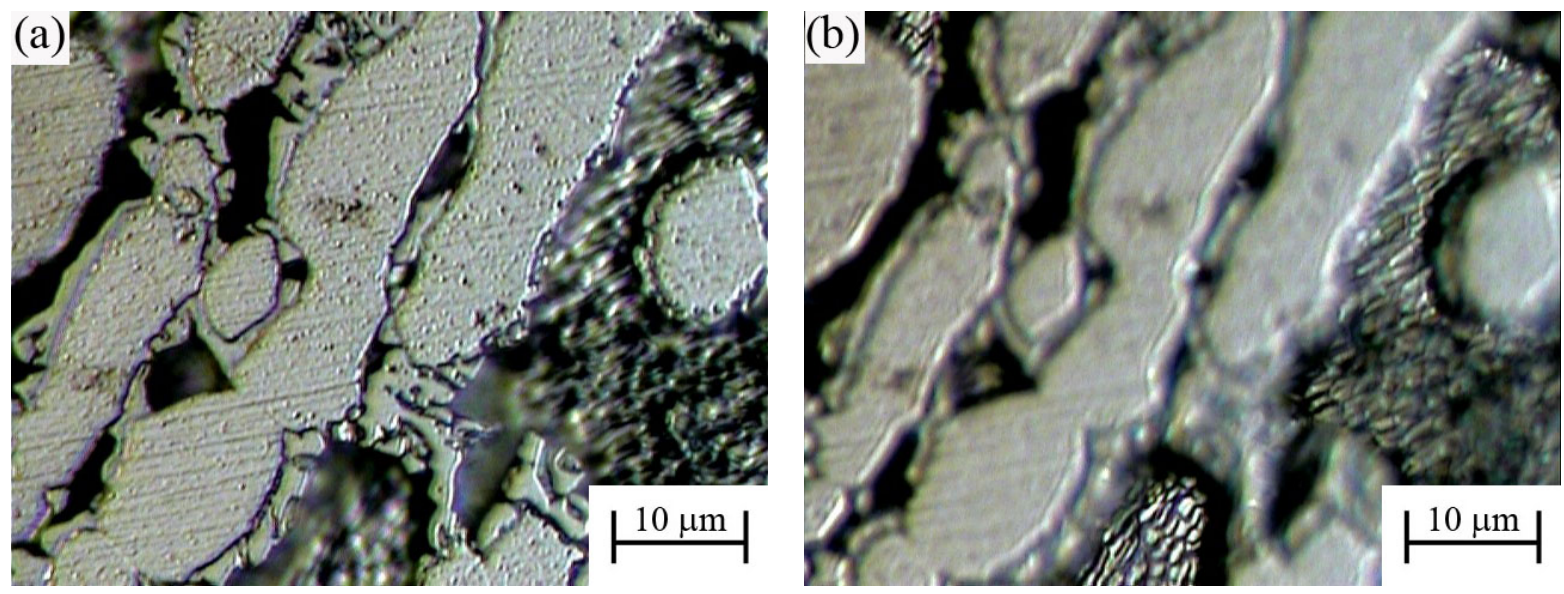

Figura 11: Análise dos pites da microestrutura após ensaios de polarização no SD-A tratado termicamente a $950{ }^{\circ} \mathrm{C}$ por 15 minutos.

Finalmente, a maioria dos valores obtidos do $E_{\mathrm{rp}}$ nos ciclos de polarização é muito baixa estando próxima do potencial de corrosão $\left(E_{c}\right)$ da liga. Este fato indica que a temperatura de repassivação da liga, nas condições estudadas, encontra-se muito abaixo dos $60^{\circ} \mathrm{C}$.

\section{CONCLUSÕES}

O presente estudo permite concluir que:

Para as condições avaliadas por tratamentos isotérmicos o potencial de pites decresce conforme a precipitação de fases deletérias, sendo o material de grão fino mais susceptível de sofrer este tipo de corrosão.

Em todos os casos, pites resultantes do ensaio de polarização cíclica foram detectados e concentrados na extinta fase ferrítica produto da transformação desta fase nos tratamentos isotérmicos efetuados.

As condições tratadas isotermicamente em diversas temperaturas por 15 minutos no SD-B apresentaram um alto valor de $\mathrm{E}_{\mathrm{p}}$. Entretanto, observou-se que quanto maior for a temperatura de tratamento, haverá uma repassivação parcial com a consequente formação de uma pequena histerese no ciclo. No caso do SD-A tratado por 15 minutos somente houve uma repassivação parcial na amostra tratada a $800{ }^{\circ} \mathrm{C}$, enquanto isso, na mesma condição, no SD-B se observou uma repassivação total, sem a existência de histerese no ciclo. Estes fatos indicam que o fenômeno de repassivação no AISD tratado isotermicamente é mais efetivo nos materiais com maior tamanho de grão.

As condições tratadas a 800 e $850{ }^{\circ} \mathrm{C}$ por 60 minutos no SD-A foram aquelas que apresentaram menores valores de $\mathrm{E}_{\mathrm{p}}$ de todas as condições avaliadas.

As condições tratadas a $950{ }^{\circ} \mathrm{C}$ por 60 minutos em ambos os materiais, apresentaram $\mathrm{E}_{\mathrm{p}}$ semelhantes, sendo estes de maior valor do que aqueles obtidos para as condições tratadas a 850 e $900{ }^{\circ} \mathrm{C}$ por 60 minutos. Os efeitos de precipitação de $\gamma_{2}$ de forma independente, a formação de placas grosseiras de fase $\sigma$ somado ao processo de redifusão na matriz, são os diferenciais nos maiores valores do $E_{p}$ obtidos nestas condições em relação às temperaturas menores de tratamento isotérmico.

Foi corroborado neste trabalho que a presença de finas lamelas de fase $\sigma$ e $\gamma_{2}$, produto da decomposição da $\delta$, são locais muito susceptíveis de quebra do filme da película passiva permitindo, deste modo, que uma corrosão seletiva desta fase prossiga facilmente. 


\section{AGRADECIMENTOS}

Os autores agradecem à CAPES, à FAPERJ e ao CNPq pelo suporte financeiro na realização deste trabalho.

\section{BIBLIOGRAFIA}

[1] MUTHUPANDI, V., SRINIVASAN, P. B., SESHADRI, S. K., et al., "Effect of weld metal chemistry and heat input on the structure and properties of duplex stainless steels welds", Materials Science and Engineering: A, v. A358, pp. 9-16, 2003.

[2] TAVARES, S.S.M., DA SILVA, M.R., NETO, J.M., "Magnetic property changes during embrittlement of a duplex stainless steel”, Journal of Alloys and Compounds, v. 313, pp. 168-173, 2000.

[3] LOPEZ, N., CID, M., PUIGGALI, M., "Influence of $\sigma$-phase on mechanical properties and corrosion resistance of duplex stainless steel”, Corrosion Science, v. 41, pp. 1615-1631, 1999.

[4] PARDAL, J.M., TAVARES, S.S.M., CINDRA FONSECA, M.P., et al., "Deleterious Phases Precipitation on Superduplex Stainless Steel UNS S32750: Characterization by Light Optical and Scanning Electron Microscopy”, Materials Research, v. 13, n. 3, pp. 401-407, 2010.

[5] RAMIREZ LONDOÑO, A.J., Estudo da Precipitação de Nitreto de Cromo e Fase Sigma por Simulação Térmica da Zona Afetada pelo Calor na Soldagem Multipassse de Aços Inoxidáveis Duplex, Dissertação de M.Sc., USP, São Paulo, Brasil, 1997.

[6] PARDAL, J.M., TAVARES, S.S.M., CINDRA FONSECA, M.P., et al., "Influence of the grain size on deleterious phase precipitation in superduplex stainless steel UNS S32750”, Materials Characterization, v. 60, pp. 165-172, 2009.

[7] ANGElini, E., DE BENEDETTI, B., ROSALBINO, F., "Microstructural Evolution and Localized Corrosion Resistance of an Aged Superduplex Stainless Steel”, Corrosion Science, v. 46, pp. 1351-1367, 2004.

[8] POTGIETER, J.H., "Influence of $\sigma$ Phase on General and Pitting Corrosion Resistance of SAF 2205 Duplex Stainless Steel”, British Corrosion Journal, v. 27, n. 3, pp. 219-223, 1992.

[9] ASTM G 61-86 (Reapproved 2003): Standard Test Method for Conducting Cyclic Potentiodynamic Polarization Measurements for Localized Corrosion Susceptibility of Iron-, Nickel-, or Cobalt-Based Alloys.

[10] RAVINDRANATH, K., MALHOTRA, S.N., "The Influence of Aging on the Intergranular Corrosion of 22 Chromium-5 Nickel Duplex Stainless Steel”, Corrosion Science, v. 37, n. 1, pp. 121-132, 1995.

[11] SRIDHAR, N., TORMOEN, G., HACKNEY, S., et al., "Effect of Aging Treatments on the Repassivation Potential of Duplex Stainless Steel S32205”, Corrosion, v. 65, pp. 650-662, 2009.

[12] SANTOS, D., MAGNABOSCO, R., "Influence of Sigma Phase Formation on Pitting Corrosion of an Aged UNS S31803 Duplex Stainless Steel”, Corrosion, v. 69, pp. 900-911, 2013.

[13] GUNN, R.N., Duplex stainless steels. Microstructure, properties and applications, Cambridge - England: Abington Publishing, 2003.

[14] Image Tool Version 3.0, Department of Dental Diagnostic Science at The University of Texas Health Science Center (UTHSCSA), San Antonio, Texas, http://ddsdx.uthscsa.edu/dig/itdesc.html. Acessado em 22 Fev. 2013.

[15] NASCIMENTO, A.M., IERARDI, M.C.F., KINA, A.Y., et al., "Pitting Corrosion Resistance of Cast Duplex Stainless Steels in 3.5\%NaCl Solution”, Materials Characterization, v. 59, pp. 1736-1740, 2008.

[16] PARDAL, J.M., TAVARES, S.S.M., CINDRA FONSECA, M.P., et al., "Influência da Precipitação de Fases Deletérias na Resistência à Corrosão por Polarização Eletroquímica de Reativação Cíclica (PERC) em Aços Inoxidáveis Superduplex”, In: Congresso Anual da ABM, Rio de Janeiro, 2010.

[17] KOBAYASHI, D.Y., WOLYNEC, S., "Evaluation of the Low Corrosion Resistant Phase Formed During the Sigma Phase Precipitation in Duplex Stainless Steels”, Materials Research, v. 2, n. 4, pp. 239-247, 1999. 
PARDAL, J.M.; TAVARES, S.S.M.; CINDRA FONSECA, M.P.; MATIAS, J.V.S., revista Matéria, v.20, n.2, pp. 374 - 383, 2015.

ERRATA

No artigo "Estudo da Influência da precipitação de fases deletérias na resistência à corrosão por ensaio de polarização anódica cíclica em aços inoxidáveis superduplex" publicado no número 2, volume 20, da revista Matéria, onde se vê:

Figura 4: Ciclos de polarização anódica das amostras tratadas isotermicamente a $800^{\circ} \mathrm{C}$ por 15 e 60 minutos nos AISD SD-A e SD-B.

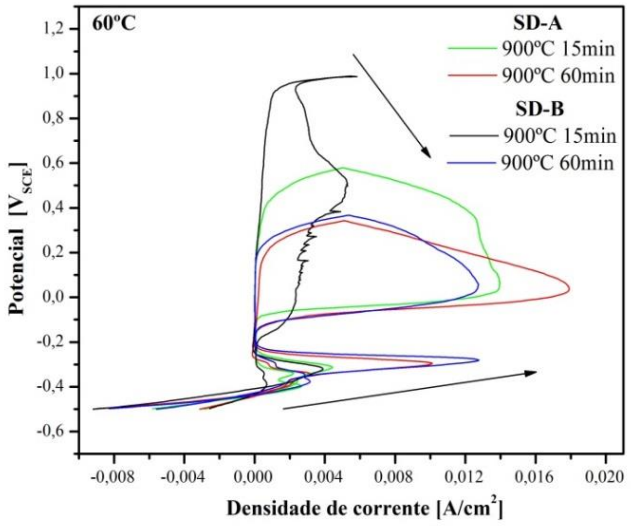

Veja-se:

Figura 4: Ciclos de polarização anódica das amostras tratadas isotermicamente a $800^{\circ} \mathrm{C}$ por 15 e 60 minutos nos AISD SD-A e SD-B.

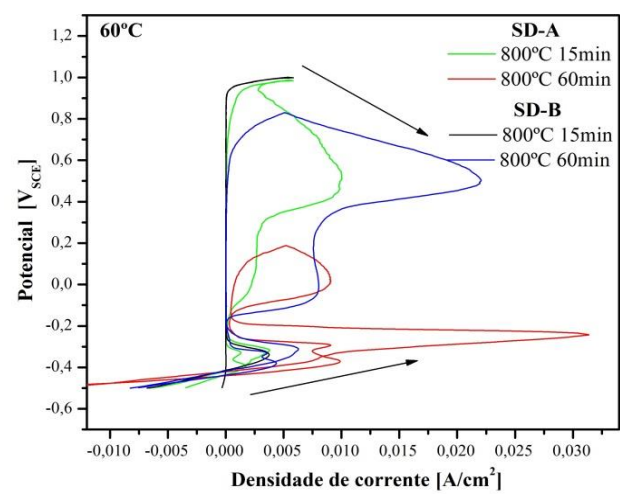

No mesmo artigo, na página 382 , onde se lê:

"Neste sentido, a Figura 10 mostra uma determinada região da microestrutura tratada isotermicamente a $950{ }^{\circ} \mathrm{C}$ por 15 minutos no SD-A." Leia-se:

"Neste sentido, a Figura 11 mostra uma determinada região da microestrutura tratada isotermicamente a $950{ }^{\circ} \mathrm{C}$ por 15 minutos no SD-A." 